\title{
CORRECTIONS
}

\section{UK academic general practice and primary care}

In this Editorial by John Campbell and colleagues (BMJ

2015;351:h4164, doi:10.1136/bmj.h4164) Health Education

England is incorrectly referred to as Higher Education England.
Cite this as: BMJ 2015;351:h4218

๑ BMJ Publishing Group Ltd 2015 